\title{
Influence of fiber aspect ratio on thermal property of composites
}

\author{
Jing Pan ${ }^{1, a, *}$, Lichun Bian ${ }^{1, b}$, Ming Gao ${ }^{1, c}$ \\ ${ }^{1}$ Key Laboratory of Mechanical Reliability for Heavy Equipments and Large Structures \\ of Hebei Province, Yanshan University, Qinhuangdao 066004, PR China \\ apanjing1234@qq.com, blcbian@ysu.edu.cn, ${ }^{\mathrm{c} g m 784135 @ 163 . c o m}$ \\ *corresponding author
}

Keywords: Thermal expansion coefficients, composites, fiber aspect ratio

\begin{abstract}
In the present investigation, a micromechanics model for predicting thermal expansion coefficients of unidirectional fiber reinforced composites is proposed, and the influence of fiber aspect ratio on the coefficients is also investigated. It is noted that the present model offers a direct prediction of thermal expansion coefficients and can account for the effect of fiber aspect ratio and volume fractions. Moreover, the thermal expansion coefficients of composites with different fiber volume fractions are very sensitive to the aspect ratio of the fiber. The present investigation has the advantage of enhancing the composite performance by adjusting the filler aspect ratio and volume fraction to obtain the desired effective material responses for engineering applications.
\end{abstract}

\section{Introduction}

In modern engineering structures, the fiber reinforced composite materials are widely used due to their some advantages, as compared with traditional materials. These fiber reinforced composite materials include a high stiffness to weight ratio, an excellent durability, and a design flexibility. Therefore, understanding the behavior of composites relative to the properties of fiber and matrix materials is desirable for practical applications. To-date, many mechanics models have been developed to predict the coefficients of thermal expansion (CTEs) of unidirectional fiber reinforced composites [1,2]. Bian and Zhao [3] proposed a continuum model to study the mechanical properties of carbon nanotubes, in which a thermal expansion coefficient of carbon nanotubes is proposed, and is defined as a continuous variation.

Gusev [4] derived an exact self-consistent solution of effective thermal expansion coefficient from an n-layered composite sphere model using the method of homogeneous solutions. Karadeniz and Dilek [5] used a representative unit cell to model the micro-structure of composites and determined the thermal expansion coefficients of composite materials using finite element method. Islam et al. [6] studied the linear thermal expansion coefficients of unidirectional composites systematically by the finite element method. Based on the composite macro-data, a combined experimental and numerical methodology for the evaluation of fiber properties was presented in the study [7]. Jarali et al [8] derived the effective electric, thermal, and moisture properties of carbon nanotube (CNT) epoxy composites by considering the agglomeration effect of CNT concentrations 
in the epoxy matrix. Dong [9] calculated the transverse CTEs of unidirectional carbon fiber composites by a finite element analysis using a representative unit cell.

The aim of the present paper is to investigate the CTEs of unidirectional fiber reinforced composites, with an emphasis on the influence of fiber aspect ratio on the CTEs. The selfconsistent and Mori-Tanaka approaches have been extended in the present investigation. It is a clearly evidence that the parameter of fiber aspect ratio has an significant effect on the longitudinal CTEs and transverse CTEs. Moreover, the CTEs of composites at different fiber volume fractions are very sensitive to the aspect ratio of fiber.

\section{Model development}

For unidirectional ellipsoid inclusion $\left(a_{1} \neq a_{2}=a_{3}\right)$ with $x_{1}$ to be the axis of rotation symmetry and plane 2-3 is isotropic, as shown in Fig. 1, and the composite is transversely isotopic materials.
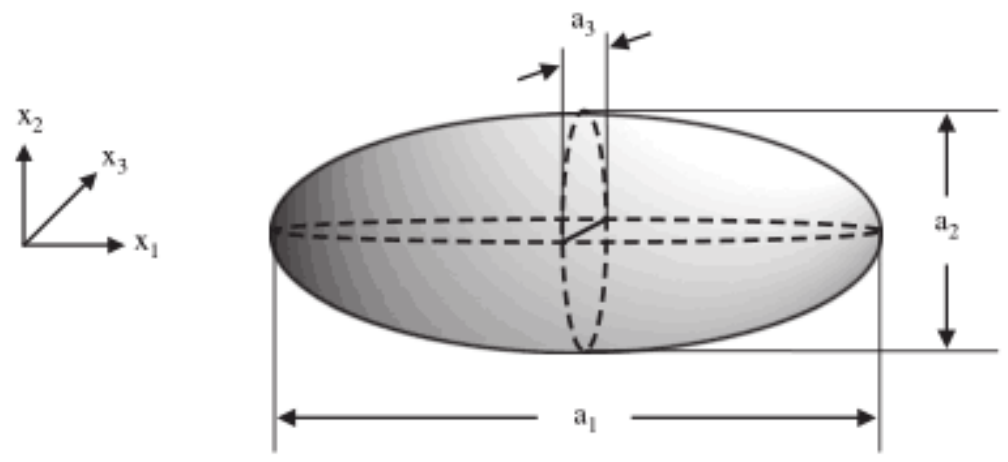

Figure 1 Coordinate system of unidirectional ellipsoid inclusion along the $x_{1}$ direction in the matrix with the aspect ratio $\lambda=a_{1} / a_{2}\left(a_{1} \neq a_{2}=a_{3}\right)$

\subsection{Basic theory}

The representative element of composites is subjected to uniform stress $\bar{\sigma}$. Local stress and strain caused in representative element are denoted by $\boldsymbol{\sigma}$ and $\boldsymbol{\varepsilon}$, respectively. In the same way, it is assumed that the composite is subjected to a uniform temperature change $\Delta \mathrm{T}$. Local stress and strain caused in representative element are denoted by $\sigma^{T}$ and $\varepsilon^{T}$, respectively. Local stress $\sigma$ and $\sigma^{T}$ conform to the balance equation.

According to the definition of the coefficient of thermal expansion:

$\left\langle\varepsilon^{T}\right\rangle=\alpha^{c} \Delta T$

For the composite material composed of two phase materials, the thermal expansion coefficients of the composite, matrix and filler are denoted by $\boldsymbol{\alpha}^{c}, \boldsymbol{\alpha}_{m}$ and $\boldsymbol{\alpha}_{f}$, respectively, and we have

$$
c_{f} \boldsymbol{\alpha}_{f}\langle\boldsymbol{\sigma}\rangle_{f}+c_{m} \boldsymbol{\alpha}_{m}\langle\boldsymbol{\sigma}\rangle_{m}=\overline{\boldsymbol{\sigma}} \boldsymbol{\alpha}^{c}=\boldsymbol{\alpha}^{c} \overline{\boldsymbol{\sigma}}
$$

where $c_{m}$ and $c_{f}$ are volume fractions of matrix and filler, $\langle\boldsymbol{\sigma}\rangle_{m}$ and $\langle\boldsymbol{\sigma}\rangle_{f}$ are stresses of matrix and filler, respectively.

The effective thermal expansion coefficient of composites can be obtained as follows

$$
\boldsymbol{\alpha}^{c}=\boldsymbol{\alpha}_{f}\left(\boldsymbol{H}_{f}-\boldsymbol{H}_{m}\right)^{-1}\left(\overline{\boldsymbol{H}}-\boldsymbol{H}_{m}\right)+\boldsymbol{\alpha}_{m}\left(\boldsymbol{H}_{m}-\boldsymbol{H}_{f}\right)^{-1}\left(\overline{\boldsymbol{H}}-\boldsymbol{H}_{f}\right)
$$


For unidirectional ellipsoid inclusion, the composite is transversely isotopic materials. Therefore, the thermal expansion coefficient of composites can be expressed as follows,

$$
\alpha_{i j}^{c}=\alpha_{i j}^{m}+\left(\alpha_{k l}^{f}-\alpha_{k l}^{m}\right)\left(H_{k l m n}^{f}-H_{k l m n}^{m}\right)^{-1}\left(\bar{H}_{m n i j}-H_{m n i j}^{m}\right)
$$

Here, considering a unidirectional ellipsoid inclusion and macroscopically isotropic composite, the longitudinal CTEs and transverse CTEs are defined by $\alpha_{11}^{c}$ and $\alpha_{22}^{c}$, respectively. The longitudinal CTE $\alpha_{11}^{c}$ and transverse CTE $\alpha_{22}^{c}$ are obtained as:

$$
\begin{aligned}
& \alpha_{11}^{c}=\alpha_{m}+\frac{\alpha_{f}-\alpha_{m}}{1 / K_{f}-1 / K_{m}}\left[\frac{3(\bar{k}-\bar{l})}{\bar{k} \bar{n}-\bar{l}^{2}}-\frac{1}{K_{m}}\right] \\
& \alpha_{22}^{c}=\alpha_{m}+\frac{\alpha_{f}-\alpha_{m}}{1 / K_{f}-1 / K_{m}}\left[\frac{3(\bar{n}-\bar{l})}{2 \bar{k} \bar{n}-2 \bar{l}^{2}}-\frac{1}{K_{m}}\right]
\end{aligned}
$$

\subsection{Elastic constants considering fiber aspect ratio}

The effective elastic modulus of hybrid inclusions and matrix can be calculated by different micromechanics methods. In this paper, it is assumed that the composites are transversely isotropic, and the elastic modulus of composites is estimated by the Mori-Tanaka approach. The basic formula of the equivalent modulus of composite according to the Mori-Tanaka approach is

$$
\overline{\mathbf{L}}=\boldsymbol{L}_{m}+c_{f}\left[\left(\boldsymbol{L}_{f}-\boldsymbol{L}_{m}\right)^{-1}+c_{m} \boldsymbol{Q}\right]^{-1}
$$

Here, the subscripts $m$ and $f$ denote the matrix and effective fibers, respectively. The components of tensor $\boldsymbol{Q}$ can be derived by $\boldsymbol{Q}=\boldsymbol{S L}_{0}^{-1}$. The components of Eshelby's equivalent tensor $\boldsymbol{S}$ can be found in reference [10].

The expression of elastic constants can be obtained as

$$
\begin{aligned}
& \bar{k}=K_{m}+\frac{1}{3} G_{m}+\frac{c_{f}}{2 \xi}\left(\frac{1}{9 K_{f}-9 K_{m}}+\frac{1}{3 G_{f}-3 G_{m}}+c_{m} Q_{1111}\right) \\
& \bar{l}=K_{m}-\frac{2}{3} G_{m}+\frac{c_{f}}{\xi}\left(\frac{1}{6 G_{f}-6 G_{m}}-\frac{1}{9 K_{f}-9 K_{m}}-c_{m} Q_{1122}\right) \\
& \bar{n}=K_{m}+\frac{4}{3} G_{m}+\frac{c_{f}}{\xi}\left[\frac{1}{6 G_{f}-6 G_{m}}+\frac{2}{9 K_{f}-9 K_{m}}+c_{m}\left(Q_{2222}+Q_{2233}\right)\right]
\end{aligned}
$$

\section{Results and discussion}

Numerical calculations were carried out on the CTEs of composites with epoxy matrix. The material properties used in the computation are those of glass/epoxy, such that $E_{m}=3.5 \mathrm{GPa}$, $E_{f}=72 \mathrm{GPa}, \gamma_{m}=0.35, \gamma_{f}=0.2, \alpha_{m}=52.5 \times 10^{-6} /{ }^{\circ} \mathrm{C}, \alpha_{f}=5 \times 10^{-6} /{ }^{\circ} \mathrm{C}$. The results are shown in Figs. 2-5, respectively.

Figs. 2 and 3 present the change of longitudinal CTE and transverse CTE with fiber volume fractions for different aspect ratio $(\lambda>1)$. As shown in Fig. 2, the longitudinal CTE $\alpha_{11}^{c}$ decreases with the increase of fiber aspect ratio. On the contrary, the transverse CTE $\alpha_{22}^{c}$ increases with the 
increase of fiber aspect ratio, as shown in Fig. 3. Furthermore, it is also observed that the effect of fiber aspect ratio on the longitudinal CTE $\alpha_{11}^{c}$ is more significant than the transverse CTE $\alpha_{22}^{c}$ when the fiber aspect ratio is $\lambda>1$.

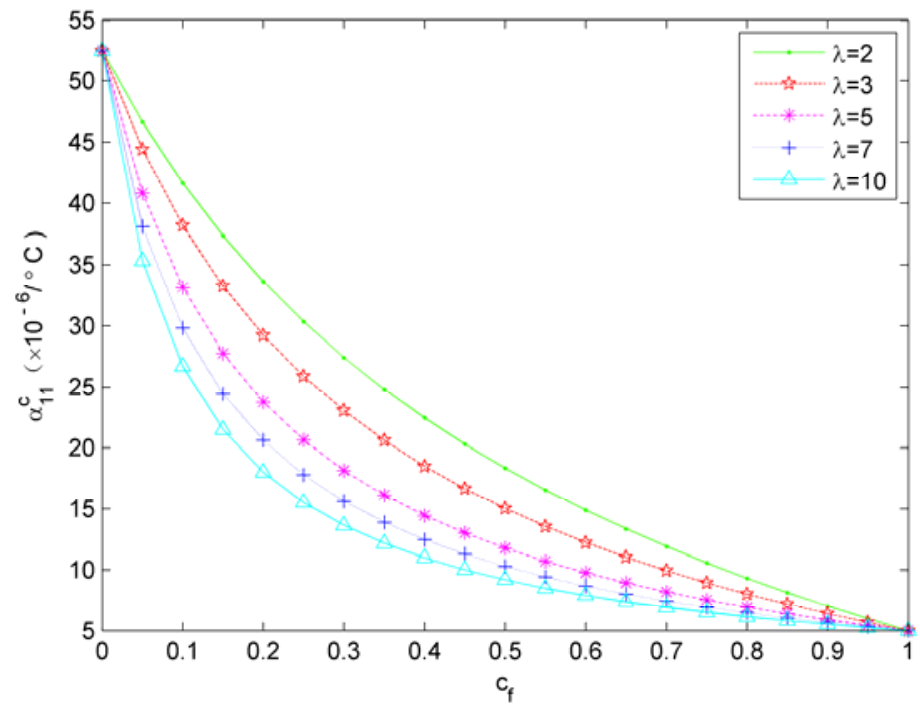

Figure 2 The change of longitudinal CTE $\alpha_{11}^{c}$ with fiber volume fractions for different aspect $\operatorname{ratio}(\lambda>1)$.

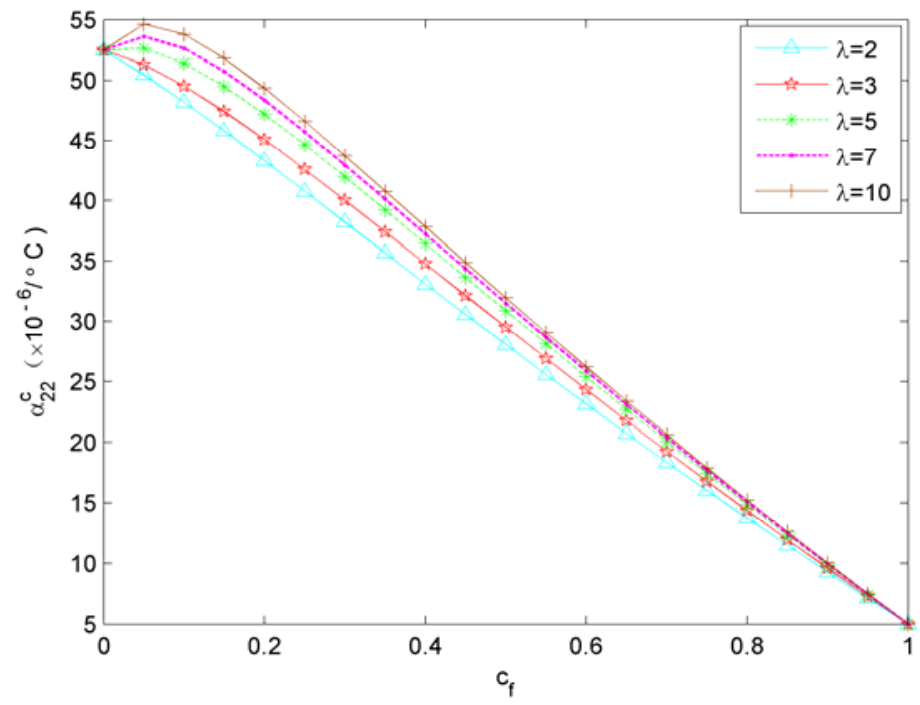

Figure 3 The change of transverse CTE $\alpha_{22}^{c}$ with fiber volume fractions for different aspect

$$
\operatorname{ratio}(\lambda>1) \text {. }
$$

To investigate the influences of fiber aspect ratio $\lambda<1$ on the CTEs of composites, the variation of CTEs with the fiber volume fractions is shown in Figs. 4 and 5. It can be seen from Fig. 4 that the longitudinal CTE increases monotonically with the increase of fiber volume fractions when $c_{f}<0.1$, whereas it decreases drastically with the increase of fiber volume fractions 
when $c_{f}>0.1$. The difference is that the transverse CTE $\alpha_{22}^{c}$ in Fig. 5 always decreases gently with the increase of fiber volume fractions in the case of aspect ratio $\lambda<1$.

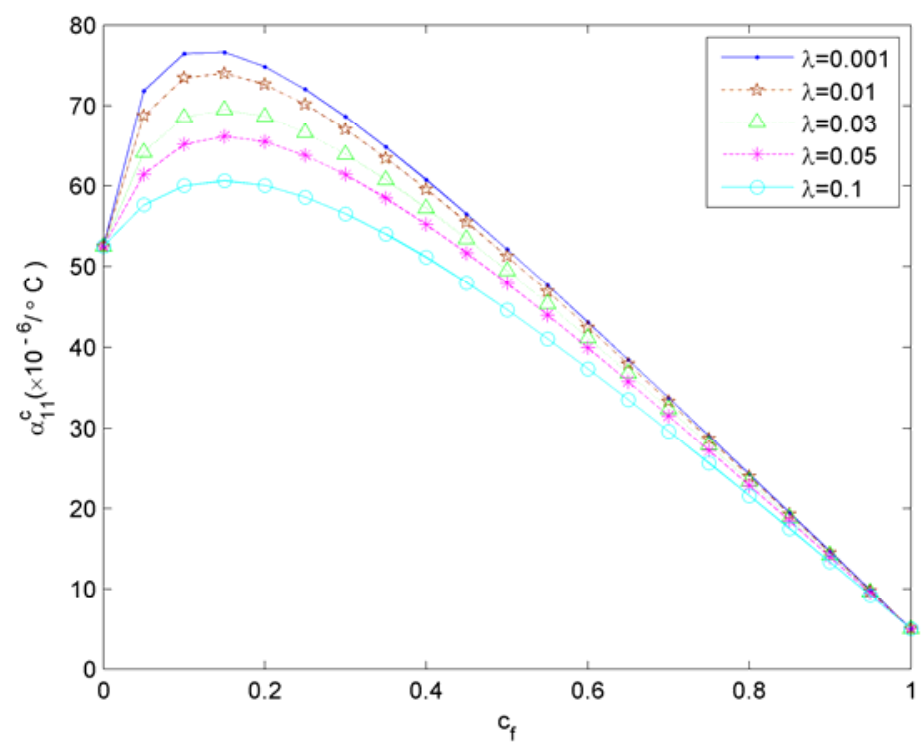

Figure 4 The variation of longitudinal CTE $\alpha_{11}^{c}$ with fiber volume fractions in the case of aspect ratio $\lambda<1$.

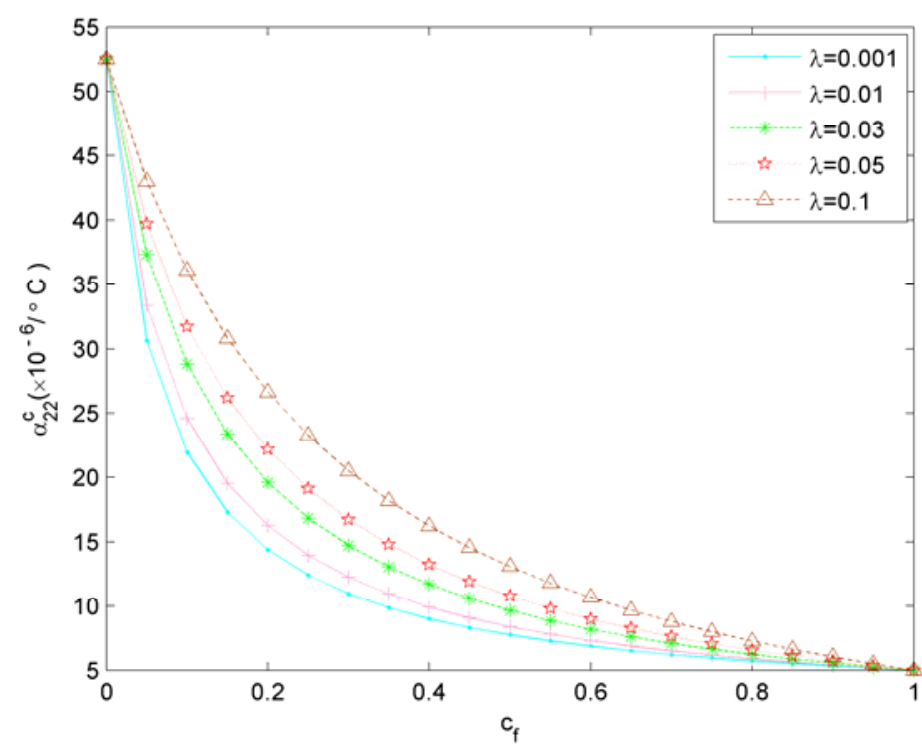

Figure 5 The variation of transverse CTE $\alpha_{22}^{c}$ with fiber volume fractions in the case of aspect ratio $\lambda<1$.

\section{Concluding remarks}

A micromechanical model is proposed for predicting thermal expansion coefficients of epoxy matrix composites. The theoretical derivations are applicable for the composites under mechanical 
or thermal environment conditions. The numerical results illustrate that the present investigation has the advantage of enhancing the composite performance by adjusting the filler aspect ratio and volume fraction to obtain the desired effective material responses.

The present model offers a direct prediction and can clearly account for the effect of fiber aspect ratio and volume fractions. It is found that other studies on CTEs available in literature are not considered the effect of filler aspect ratio. Therefore, the present analysis provides a helpful tool for optimizing material design and manufacture of composites to maintain the performance of material in serve and the production of advanced composites in engineering applications.

\section{Acknowledgements}

This research was funded by the Science Research Foundation of Hebei Advanced Institutes (ZD2017075) and Graduate Innovation Foundation of Hebei Province (CXZZBS2017053).

\section{References}

[1] Chakrabarty, A., Çağın, T. (2014) Thermo-mechanical properties of a piezoelectric polyimide carbon nanotube composite: Assessment of composite theories. Computational Materials Science, 92, 185-191.

[2] Romero P.A., Anciaux, G., Molinari, A., Molinari, J.F. (2013) Insights into the thermo-mechanics of orthogonal nanometric machining. Computational Materials Science, 72, 116-126.

[3] Bian, L.C., Zhao, H.C. (2015) Elastic properties of a single-walled carbon nanotube under a thermal environment. Composite Structures, 121, 337-343.

[4] Gusev, A.A. (2014) Effective coefficient of thermal expansion of n-layered composite sphere model: Exact solution and its finite element validation. International Journal of Engineering Science, 84, 54-61.

[5] Karadeniz, Z.H., Kumlutas, D. (2007) A numerical study on the coefficients of thermal expansion of fiber reinforced composite materials. Composite Structures, 78, 1-10.

[6] Islam, M.R., Sjölind, S.G., Pramila, A. (2001) Finite element analysis of linear thermal expansion coefficients of unidirectional cracked composites. Journal of Composite Materials, 35(19), 1762-1776.

[7] Rupnowski P, Gentza M, Sutterb J.K., Kumosa M. (2005) An evaluation on the elastic properties and thermal expansion coefficients of mediumthe elastic properties and thermal expansion coefficients of medium and high modulus graphite fibers. Composites Part A, Applied Science \& Manufacturing. 36(3), 327-338.

[8] Jarali, C.S., Patil, S.F., Pilli, S.C. (2014) Hygro-Thermo-Electric Properties of CNT Epoxy Nanocomposites with Agglomeration Effects. Journal of Applied Mechanics, 22(6), 428-439.

[9] Dong, C.S. (2009) Development of an Engineering Model for Predicting the Transverse Coefficients of Thermal Expansion of Unidirectional Fiber Reinforced Composites. Journal of Engineering Materials and Technology, 131, 0310011-0310017.

[10] Lu, P. (2013) Further studies on Mori-Tanaka models for thermal expansion coefficients of composites. Polymer, 54, 1691-1699. 